\title{
Structure and Function of Animal Cryptochromes
}

\author{
N. ÖzTÜRK, ${ }^{*}$ S.-H. Song, ${ }^{*}$ S. ÖzGÜR, ${ }^{*}$ C.P. Selby, ${ }^{*}$ L. Morrison, ${ }^{*}$ C. Partch, ${ }^{\dagger}$ \\ D. ZHONG, ${ }^{+}$AND A. SANCAR* \\ *Department of Biochemistry and Biophysics, University of North Carolina School of Medicine, Chapel Hill, \\ North Carolina 27599; 'Department of Biochemistry, University of Texas Southwestern Medical Center, \\ Dallas, Texas 75390; Departments of Physics, Chemistry, and Biochemistry, Programs of Biophysics, \\ Chemical Physics, and Biochemistry, Ohio State University, Columbus, Ohio 43210
}

\begin{abstract}
Cryptochrome (CRY) is a photolyase-like flavoprotein with no DNA-repair activity but with known or presumed blue-light receptor function. Animal CRYs have DNA-binding and autokinase activities, and their flavin cofactor is reduced by photoinduced electron transfer. In Drosophila, CRY is a major circadian photoreceptor, and in mammals, the two CRY proteins are core components of the molecular clock and potential circadian photoreceptors. In mammals, CRYs participate in cell cycle regulation and the cellular response to DNA damage by controlling the expression of some cell cycle genes and by directly interacting with checkpoint proteins.
\end{abstract}

\section{INTRODUCTION}

CRYs are FAD-based blue light photoreceptors that control growth and development in plants and the circadian clock in animals and possibly in plants (Cashmore 2003; Lin and Shalitin 2003; Sancar 2003, 2004). CRYs have sequence and structural similarities to DNA photolyases (Fig. 1), which repair UV-induced DNA damage by a photoinduced cyclic electron transfer reaction (Sancar 2003; Kao et al. 2005). These two seemingly unrelated phenomena, circadian rhythm and DNA repair, may have had a common evolutionary origin (Pittendrigh 1993; Sancar 2000; Gehring and Rosbash 2003; Lowrey and Takahashi 2004). According to this "escape from light" hypothesis, in the distant past when more UV reached the surface of the earth, an aquatic organism used a blue-light photoreceptor (CRY) to restrict its S phase to the dark phase of the day (night) so as to minimize the harmful effects of DNA damage and to regulate the organism's vertical movement to and away from the surface of the water with daily (circadian) periodicity, thereby optimizing nutrient uptake and minimizing the extent of DNA damage. This same photoreceptor may

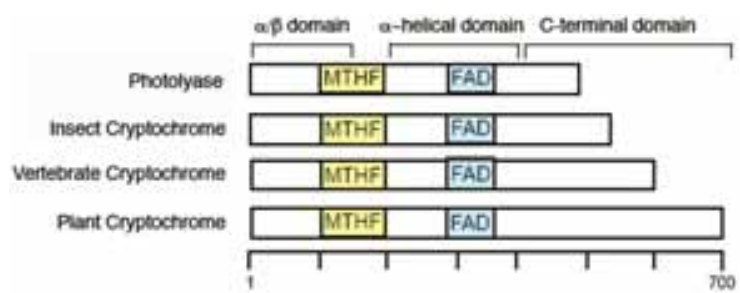

Figure 1. Schematic representation of the photolyase/CRY family proteins. The enzymes are 500-700 amino acids in length and have a modular structure with an amino-terminal $\alpha / \beta$ domain and a carboxy-terminal $\alpha$-helical domain. In addition, most CRYs have carboxy-terminal extensions ranging in size from 40 to 250 amino acids. Representative examples of the major classes are shown. The insect CRY in this figure is Insect Type 1 CRY. The approximate binding sites of the two cofactors MTHF and FAD are indicated. have also been used to repair DNA damage (photolyase) that inevitably occurred under such conditions, especially in the early days of life on earth when more UV reached the surface because of the lack of the protective ozone layer. Blue light is best suited for both tasks since only blue light can penetrate to substantial depths in water. Consequently, this hypothesis suggests that the blue light photoreceptor carrying out these two functions diverged to give rise to the present-day photolyases and CRYs. It is conceivable that future research may uncover the "missing link" of the theory: a blue light photoreceptor with both circadian and DNA-repair functions.

\section{HISTORICAL PERSPECTIVE}

Since the second half of the 19th century, plant biologists have known that blue light has a profound effect on growth, development, and phototropic movement of plants (Darwin 1881). However, efforts to identify the blue light photoreceptor initiating these responses were futile for a long period, and some plant biologists used the term "cryptochrome" as a generic name for this mysterious photoreceptor. The following explanation was given as a justification of the name: "The pigment system(s) responsible for many of the photoprocesses (as ascertained by action spectra) has been nicknamed 'cryptochrome' because of its importance in cryptogamic plants and its cryptic nature. This term, despised by many, will suffice us here just because it is shorter than other terms used, such as 'blue (UV) light photoreceptor,' and it will be a useful term until the pigments are identified" (Gressel 1977). Later work identified at least four classes of flavoproteins that mediate blue light responses in plants (Banerjee and Batschauer 2005): photolyase, the HY4 protein, phototropin, and the ZTL/ADO family. Of these, the photolyase was the first blue light photoreceptor to be identified as a flavoprotein in bacteria and many other species and has been extensively characterized (Sancar 2003). When the Arabidopsis thaliana HY4 gene, known to be required for inhibition of hypocotyl growth 
in response to blue light (Koornneef et al. 1980), was isolated and sequenced, it revealed high sequence homology with Escherichia coli photolyase, and hence, it was in retrospect, correctly speculated that HY4 encoded a blue light photoreceptor and not a signal transducer involved in blue light response (Ahmad and Cashmore 1993). Later, this protein was named cryptochrome 1 (Lin et al. 1995, 1998), and a second Arabidopsis protein that has high homology with CRY1 identified by genomics was named CRY2 (Lin and Shalitin 2003). The role of CRY in Arabidopsis growth (CRY1) and differentiation (CRY2) was well established by 1995 (see Guo et al. 1998). However, as late as 1997, it was thought that CRY had no role in circadian photoreception in plants (Millar and Kay 1997).

The first report implicating CRY in the circadian clock of any organism, plant or animal, came about from the study of DNA repair, in particular, the repair of UV damage in humans by photolyase. This issue had been controversial for nearly 25 years when Li et al. (1993) conducted an exhaustive study with a highly specific and sensitive assay, concluding that humans, like all placental mammals, lacked photolyase (Li et al. 1993). However, a 1995 release of a human expressed sequence tag (EST) list contained a "photolyase ortholog" entry (Adams et al. 1995). In light of this finding and the discovery of a photolyase in Drosophila and rattlesnake (Todo et al. 1993, 1996; Kim et al. 1996) that repairs the minor UV-induced lesion, the (6-4) photoproduct, in contrast to the classic photolyase that repairs cyclobutane pyrimidine dimers (CPDs), the earlier conclusion regarding the lack of photolyase in humans needed reevaluation. This was done by Hsu et al. (1996) who, in addition to the "photolyase ortholog" in public databases, discovered a second human photolyase gene. Human cells expressing both genes and recombinant proteins encoded by both genes were tested for CPD and (6-4) photolyase activities and were found to lack both. Moreover, the proteins encoded by these genes, like most photolyases (Johnson et al. 1988) and Arabidopsis CRY (Lin et al. 1995; Malhorta et al. 1995), contained FAD (flavin-adenine dinucleotide) and a pterin cofactor. Therefore, it was concluded that these proteins were not repair enzymes but that, like Arabidopsis CRYs, they performed non-repair-related blue light functions and were named human CRY1 and 2 (Hsu et al. 1996). In humans and most other animals, the two well-characterized, lightmediated reactions are vision and circadian entrainment. Because opsins are securely established as the vision pigment, it was suggested that the human CRYs might be circadian photoreceptors (Hsu et al. 1996; Zhao and Sancar 1997) and experiments were set up to test this prediction in the mouse.

The first experimental data linking CRY to the circadian clock was the finding that mouse CRY1 was highly expressed in the suprachiasmatic nucleus (SCN) (Fig. 2A) with an expression pattern that exhibited periodicity with a peak at ZT8 and nadir at ZT20 (Miyamoto and Sancar 1998, 1999). The functional proof that the mammalian CRY controlled the circadian clock came shortly afterward when $m C r y 2^{--}$mice were generated and tested for circadian phenotypes (Fig. 2B). It was found that the mutant mice had a period about 1 hour longer than that of wild-type littermates and the CRY2 knockout exhibited greatly increased phase-shifts in response to light pulses (Thresher et al. 1998). These data firmly established CRY as a core clock protein regardless of its potential involvement in circadian photoreception. Nearly simultaneously, the strongest evidence to date for a circadian photoreceptive role of CRY in animals was obtained when it was discovered that a Drosophila mutant selected for reduced circadian photosensitivity contained a missense mutation in the Drosophila ortholog of CRY, DmCry (Emery et al. 1998; Stanewsky et al. 1998). In light of these developments, the Arabidopsis CRY mutants were tested for circadian photoreception, and it was found that under a particular lighting regimen, Arabidopsis CRYs also

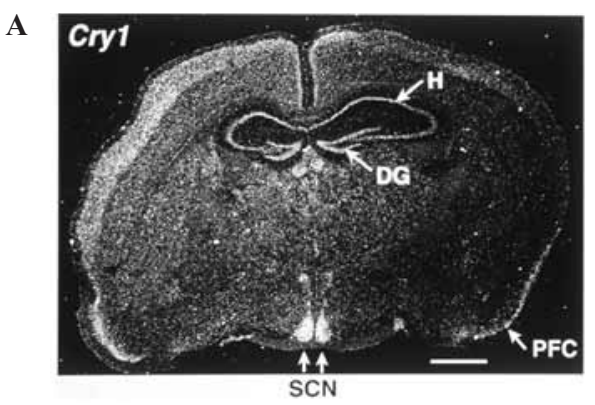

B

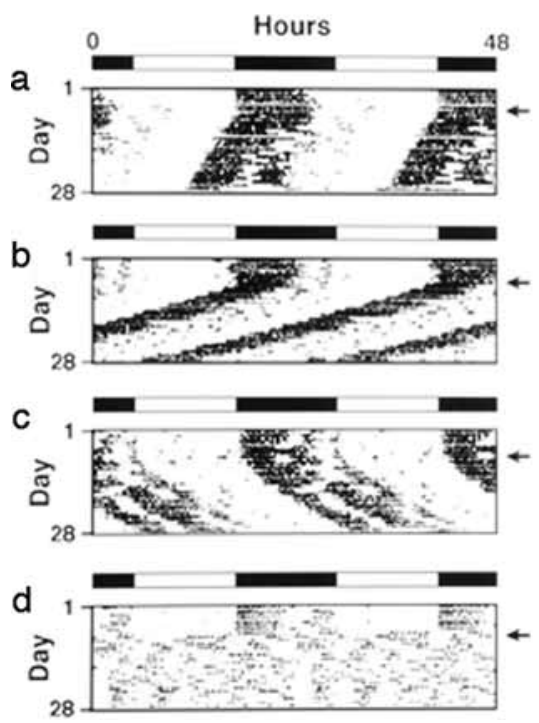

Figure 2. First experimental data implicating CRYs in the circadian clock. (A) Expression of mammalian CRY1 in the mouse $\mathrm{SCN}$. Expression was measured by in situ hybridization. This sample was prepared at ZT6 when mammalian CRY1 expression is at its zenith; at ZT18 (or CT18) mammalian CRY1 is virtually undetectable. (SCN) Suprachiasmatic nucleus; (PFC) piriform cortex; (DG) dentate gyrus; (h) hippocampus. ( $B$ ) Effect of CRY mutation on mouse circadian behavior. The locomotor activities of wild-type and mutant mice were recorded for 28 days. At the day indicated by arrows, the animals were switched from LD to DD conditions. (a) Wild-type: $\tau=23.7 \mathrm{hr}$; (b) Cry $1^{-1-}: \tau=22.7$

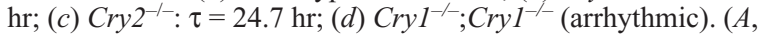
Reprinted, with permission, from Miyamoto and Sancar 1998; $B$, reprinted, with permission, from Vitaterna et al. 1999 [both (C) National Academy of Sciences].) 
appeared to participate in circadian photoreception under a particular lighting regimen (Somers et al. 1998). Finally, mouse Cry $1^{-1-} ; \mathrm{Cry}^{-/-}$mutants were constructed and found to have lost circadian rhythm entirely (van der Horst et al. 1999; Vitaterna et al. 1999), thus expanding the conclusion based on the Cry $2^{-1-}$ mutant and consolidating the role of CRYs as core clock proteins in mammals (Fig. 2B). These results set the stage for the findings that mammalian CRYs interacted with all core clock proteins as revealed by yeast two-hybrid assay (Ceriani et al. 1999) and that mammalian CRYs acted as potent repressors of the CLOCK/BMAL1 transcriptional activator complex as revealed by reporter gene assays (Kume et al. 1999) and by the constitutive, elevated expression of mPer genes in $\mathrm{Cry}^{-/-} ; \mathrm{Cry}^{-/-}$mice (Vitaterna et al. 1999) and eventual development of transcriptional-translational feedback loop (TTFL) model for animal circadian clock (Gekakis et al. 1998; Young and Kay 2001; Reppert and Weaver 2002). As is apparent from the summary of the field given above, the identification of CRYs as circadian proteins followed a conventional and inductive scientific approach and was not, as suggested, "accidental" (Hunt and Sassone-Corsi 2007).

\section{PHYLOGENY AND FUNCTIONAL CLASSIFICATION}

To assess the evolution of the photolyase/CRY family, we performed an exhaustive search of annotated sequence databases, retrieving more than 250 sequences of photolyases and CRYs from all three kingdoms of life. Phylogenetic analysis by neighbor-joining and maximum parsimony methods grouped these sequences into eight major classes. A reduced tree with gene names is shown in Figure 3. Seven of these classes have been previously described and functionally characterized to varying degrees: class I and class II CPD photolyases, (6-4) photolyase, single-stranded DNA photolyase (previously called DASH CRYs), and plant, insect, and vertebrate CRYs. However, this analysis led to two unexpected findings: First, a group of novel independently segregating bacterial sequences was identified, comprising a new class (class III CPD photolyase), and second, vertebratelike CRY sequences were discovered in nondrosophiloid insects. In vivo photoreactivation data on a class III CPD photolyase from Caulobacter crescentus indicate that this is indeed a CPD photolyase (see Partch 2006). Second, as first reported by Zhu et al. (2005) and Yuan et al. (2007), several nondrosophiloid insects such as mosquito, honeybee, and silkmoth possess a vertebrate-like CRY, suggesting that the origin of vertebrate CRY predates the last common ancestor shared by insects and vertebrates. The name of bilateral CRYs was proposed because they are present in at least two branches of Bilateria, animals defined by bilateral symmetry (Partch 2006). However, since the names of "Insect CRY1" and "Insect CRY2" have been used for the drosophiloid and vertebrate-type CRYs (Zhu et al. 2005; Yuan et al. 2007), we will follow that nomenclature. Current data indicate that Insect CRY1s are sensitive to photoinduced degradation in vivo, whereas Insect CRY2s are not, but instead, they function

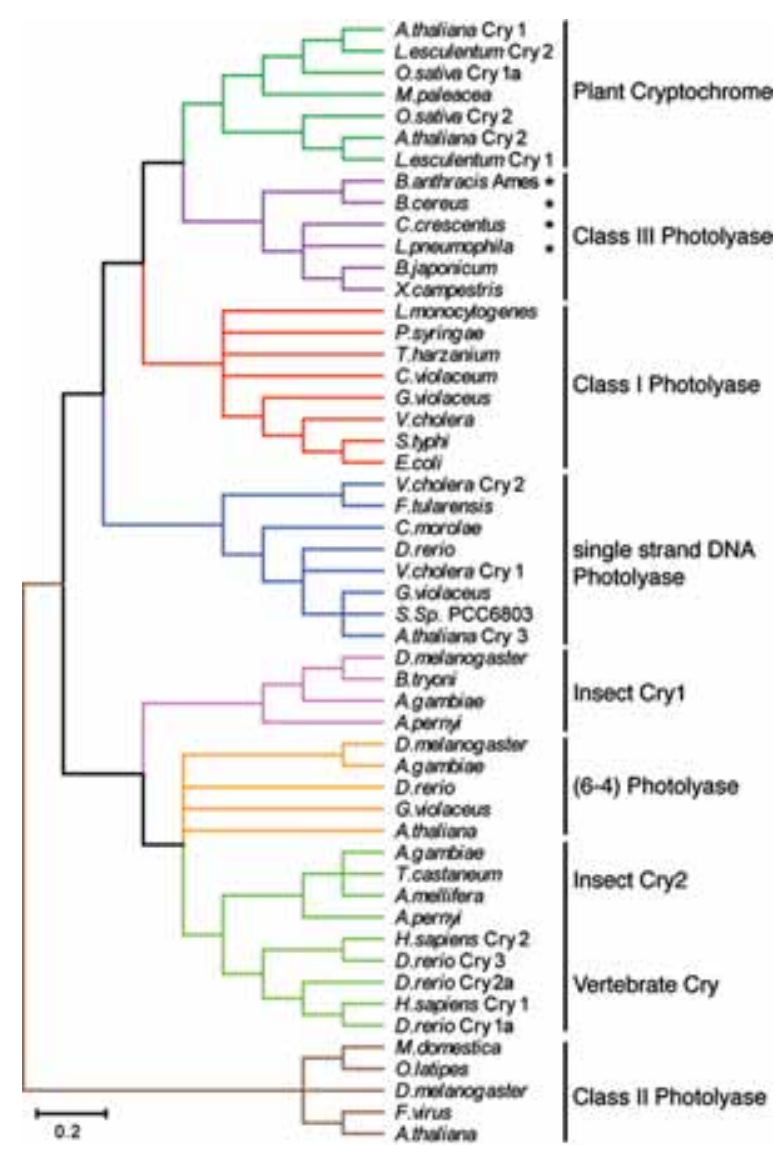

Figure 3. Evolutionary relationships in the photolyase/CRY family. Annotated photolyase and CRY sequences $(>250$ total sequences) from GenBank and Swiss-Prot databases were aligned using ClustalW. Alignments were manually verified, and an unrooted phylogenetic tree was generated using neighbor-joining methods (MEGA 4.1). Phylogenetic analysis of representative sequences are shown. Eight major classes are identified, including a novel group of photolyases (Class III, purple) that is a sister taxon to plant CRYs (green). The singlestranded DNA photolyase class was previously misclassified as CRY with Cry-DASH designation. However, recent work has shown that these are CPD photolyases specific for singlestranded DNA (Selby and Sancar 2006). Asterisks indicate organisms with sequenced genomes that possess this single photolyase gene and a literature report of photoreactivation. Bar represents residue substitutions per site.

as repressors of the Clock/Cycle complex. Drosophila possesses only Insect CRY1, the honeybee Apis mellifera has only Insect CRY2, and the monarch butterfly Danaus plexippus has both. It should also be noted that the assignment of the insect CRY1s as solely photoreceptors and the Insect CRY2/vertebrate CRY family exclusively as repressors is not universally accepted. There is credible evidence that DmCRY functions as a repressor (Collins et al. 2006). Similarly, there is considerable genetic evidence that vertebrate CRYs, in addition to the repressor activity, may function as photoreceptor/phototransducers (Thresher et al. 1998; Selby et al. 2000; Thompson et al. 2003; Tu et al. 2004). In addition to this phylogenetic classification, the photolyase/CRY family can be divided into functional classes. These classifications do not nec- 
essarily converge in all facets as evidenced by the fact that class I and class II CPD photolyases are more phylogenetically distant from each other than class I photolyases and plant CRYs, yet class I and class II photolyases perform exactly the same repair function.

\section{STRUCTURES OF PHOTOLYASE AND CRYPTOCHROME}

Photolyase/CRY family proteins are $50-80-\mathrm{kD}$ proteins of 500-700 amino acids in length with two chromophores/cofactors (Fig. 4). One of the cofactors is always FAD, serving as the catalytic cofactor. The other cofactor serves as a photoantenna and is most commonly methenyltetrahydrofolate (MTHF) or, in rare instances, 8hydroxy-5-deazaflavin (8-HDF) in organisms that synthesize this chromophore (Sancar 2003; Partch and Sancar 2005). Recently, it was reported that the Thermus thermophilus photolyase contains flavin mononucleotide (FMN) (Ueda et al. 2005) and photolyase from Sulfolobus tokodaii contains FAD (Fujihashi et al. 2007) as the second chromophore, suggesting that photolyase is capable of utilizing a variety of chromophores as photoantenna. Because of the high sequence and structural similarities

$\mathbf{A}$

\section{E.coli Phr}
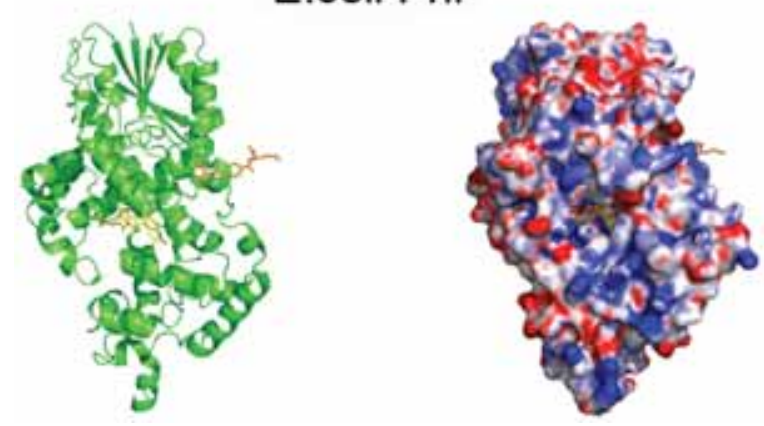

B
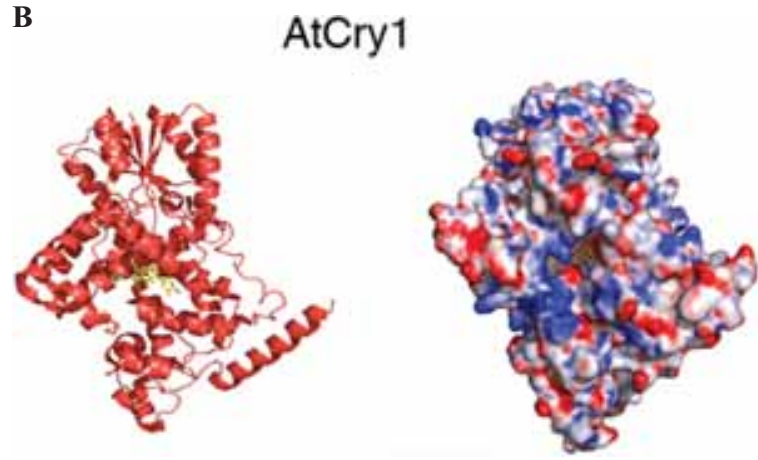

Figure 4. Crystal structures of the photolyase/CRY family. Both ribbon diagram and surface potential representations are shown. $(A)$ E coli photolyase; $(B) A$. thaliana CRY1-PHR domain. Note that the overall architectures are very similar including the hole leading to the FAD cofactor in the core of the $\alpha$-helical domain. However, although photolyase possesses a positively charged DNA-binding groove running the length of the surface, this groove is mostly lined with negatively charged residues in AtCRY1. Note also that the crystal structure of AtCRY1 is lacking the MTHF cofactor. between photolyase and CRY, it is generally assumed that CRYs have the same two cofactors as well. However, no CRY has been purified to date from its native source and those that have been purified as recombinant proteins contain FAD to varying levels and either trace amounts of MTHF or none at all (Lin et al. 1995; Malhotra et al. 1995; Özgür and Sancar 2003; Song et al. 2007). Hence, formal proof that CRYs contain MTHF, or any other secondary chromophore, is lacking.

CRYs diverge from photolyases in another significant aspect: Nearly all CRYs (but not CRY1 of Sinapis alba) possess carboxy-terminal domains beyond that of the photolyase homology region (PHR) ranging from 30 to 350 amino acids in length. Of significance, the sequences of these carboxy-terminal domains are not conserved from plants to animals (Partch and Sancar 2005). Biochemical and biophysical tests show that the carboxy-terminal domains of CRYs are highly unstructured when expressed alone (Partch et al. 2005; Kottke et al. 2006) but assume a rigid structure by interacting with the PHR domain (Lin and Shalitin 2003; Partch et al. 2005). Light-induced conformational change from order to disorder in AtCRY1 has been proposed to initiate the photosignaling reaction (Partch et al. 2005; Kottke et al. 2006; Yu et al. 2007).

Crystal structures of several photolyases are available and are quite similar (Huang et al. 2006; Fujihashi et al. 2007). In contrast, only the PHR domain of AtCRY1 has been crystallized (Brautigam et al. 2004). The structures of photolyases are characterized by two modular domains (Fig. 4): an amino-terminal $\alpha / \beta$ domain and a carboxyterminal $\alpha$-helical domain connected by a long interdomain loop. The catalytic FAD chromophore is bound within the $\alpha$-helical domain in an unusual U-shaped conformation, with the isoalloxazine ring held in close proximity to the adenine ring, and the second chromophore is bound in a cleft located between the two domains close to the surface of the protein. Surface potential representation of the photolyase structure reveals a positively charged DNA-binding groove running the length of the molecule. A hole of approximately $10 \AA$ in diameter, located in the middle of this groove, allows access of solvent and oxygen to the FAD molecule. Additionally, this hole is of the right dimensions and polarity to allow entry of a pyrimidine dimer to within van der Waals contact distance of the isoalloxazine ring of FAD. The structure of AtCRY1 PHR domain structure is very similar to that of photolyase in many aspects, including the substrate-binding cavity; however, AtCRY1 lacks the positively charged DNAbinding groove, and in fact, many of the amino acid residues lining this groove are negatively charged which may partly be responsible for the lack of DNA-repair activity by plant CRYs (Brautigam et al. 2004). The other significant aspect of the AtCRY1 PHR crystal structure is the lack of MTHF or any other second chromophore.

\section{REACTION MECHANISM OF PHOTOLYASE}

Currently, three classes of flavin-based blue light photoreceptors are known, each utilizing flavin in different chemical forms or oxidation states (Banerjee and Batschauer 2005; Losi 2007): LOV proteins (FMN), 


\section{STRUCTURE AND FUNCTION OF ANIMAL CRYPTOCHROMES}

BLUF proteins (FAD), and CRY/photolyase $\left(\mathrm{FADH}^{-}\right)$. Blue light causes the formation of a blue-shifted FMNcysteine $\mathrm{C}(4 \mathrm{a})$-thioladduct $\left(\lambda_{\max }=340 \mathrm{~nm}\right)$ in LOVdomain-containing proteins such as phototropin and hydrogen-bond rearrangement of FAD and neighboring amino acids that causes a red-shift in FAD absorption (from between 365 and 445 to 371 and $460 \mathrm{~nm}$ ) in BLUFdomain-containing proteins such as the Euglena gracilius photoactivated adenylyl cyclase (PAC). These could be potential models for the CRY photocycle. However, because of its evolutionary and structural relatedness to photolyase, CRY is more likely to have a photocycle similar to that of photolyase. For this reason, the photolyase photocycle is discussed below in some detail (Fig. 5).

Photolyase recognizes the $30^{\circ}$ kink caused in DNA by a cyclobutane pyrimidine dimer (Pyr $<>$ Pyr), and it flips out the dimer from within the duplex to the active site cavity of the enzyme to form a highly stable E•S complex. Light initiates catalysis: The MTHF (or 8-HDF) photoantenna absorbs a photon and transfers the energy to $\mathrm{FADH}^{-}$(the active form of flavin in photolyase) by Förster resonance energy transfer. The excited state flavin, ${ }^{1}\left(\mathrm{FADH}^{-}\right)^{*}$, transfers an electron to $\mathrm{Pyr}<>\mathrm{Pyr}$ to generate a charge-separated radical pair $\left(\mathrm{FADH}^{\circ}-\mathrm{Pyr}<>\mathrm{Pyr}^{\circ-}\right)$. The cyclobutane ring is split by cycloreversion and the flavin radical is restored to the catalytically competent $\mathrm{FADH}^{-}$form by back electron transfer following splitting of the cyclobutane ring. Significantly, at the end of the catalytic cycle, there is no change in the redox state of flavin or the substrate/product and hence the repair reaction is not a redox reaction. Following catalysis, the repaired dinucleotide no longer fits in the active-site pocket and is ejected back into the duplex, and the repaired DNA dissociates from the enzyme. It is thought that the (6-4) photolyase employs essentially the same mechanism as classical photolyase (Sancar 2003; Li et al. 2006).
A
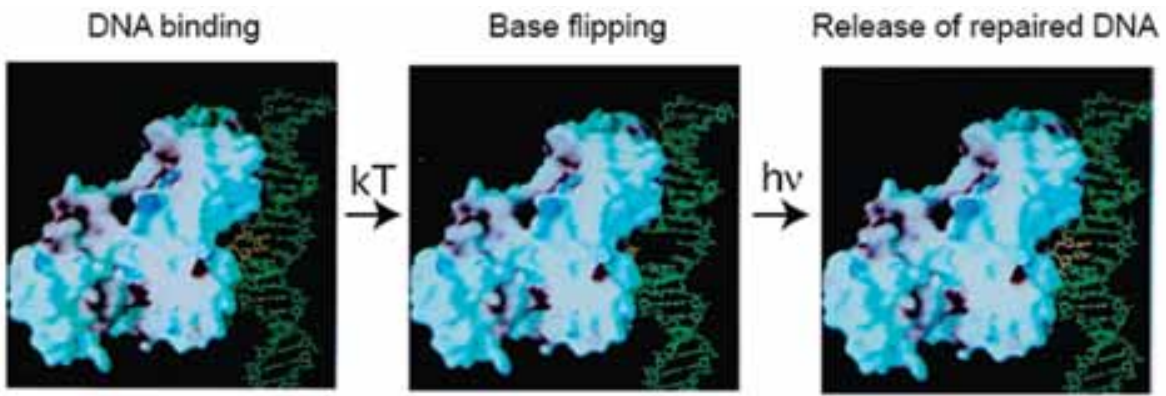

B

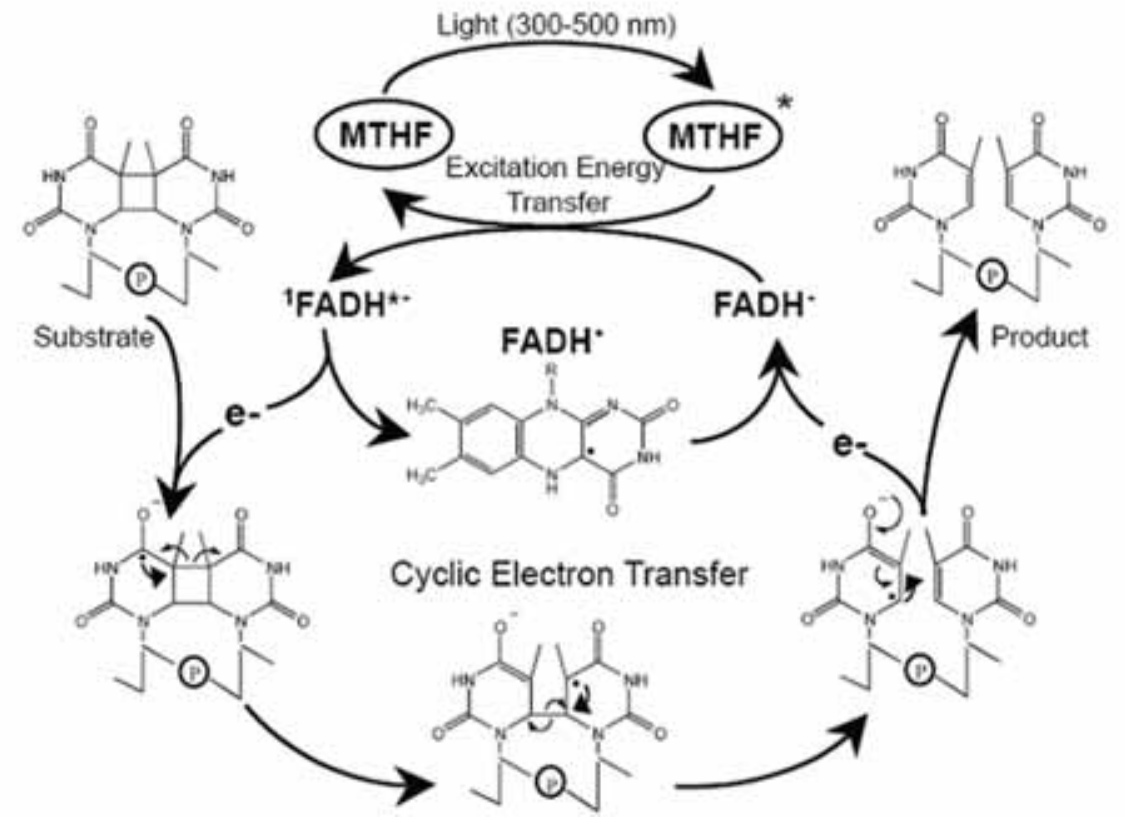

Figure 5. Reaction mechanism of DNA photolyase. (A) Substrate binding (dark reaction) and product release. The enzyme binds the DNA backbone around the cyclobutane pyrimidine dimer by random collision, forming a low-affinity complex. Then, it flips out the dimer into the active site cavity. The enzyme-substrate complex formation is a thermal $(k T)$ reaction, independent of light. Following the light $(h v)$ reaction, the repaired dinucleotide is ejected from the active-site cavity and DNA dissociates from the enzyme. $(B)$ Catalysis (light reaction). The photoantenna (MTHF) absorbs a photon and transfers the excitation energy to $\mathrm{FADH}^{-}$by FRET. The ${ }^{1}\left(\mathrm{FADH}^{-}\right)^{*}$ then transfers an electron to the cyclobutane dimer to generate a radical pair of flavin and pyrimidine dimer. The cyclobutane ring is split, and the electron returns to $\mathrm{FADH}^{\circ}$ to regenerate catalytically active $\mathrm{FADH}^{-}$. The reaction is a cyclic redox reaction with no net change in the redox status of either the enzyme or the substrate/product at the end of the reaction. 
Finally, a comment should be made on the action spectra of photolyases. An action spectrum is a plot of the rate of a photochemical or photobiological reaction as a function of the wavelength eliciting the reaction (see Sancar 2000). Although the catalytic cofactor in photolyases is $\mathrm{FADH}^{-}$ with $\lambda_{\text {max }} \sim 360 \mathrm{~nm}$ and molar extinction coefficient $\varepsilon$ $\sim 6000 \mathrm{M}^{-1} \mathrm{~cm}^{-1}$, the action spectra of photolyases are dominated by the photoantennas that have much higher extinction coefficients and $\lambda_{\max }$ at longer wavelengths. Thus, in photolyases with MTHF cofactor $\left(\varepsilon=25,000 \mathrm{M}^{-1} \mathrm{~cm}^{-1}\right)$ and $\lambda_{\text {max }}=380-415 \mathrm{~nm}$ (depending on the enzyme), the action spectra match that of MTHF absorption spectra. Similarly, in photolyases with 8-HDF as the second chromophore $(\varepsilon$ $=40,000 \mathrm{M}^{-1} \mathrm{~cm}^{-1}$ and $\left.\lambda_{\max }=440 \mathrm{~nm}\right)$, the action spectrum maximum is at $440 \mathrm{~nm}$. It should be noted that photolyases can carry out photorepair in the absence of the second chromophore because $\mathrm{FADH}^{-}$can directly absorb a photon and initiate catalysis. In this case, the action spectrum matches the absorption spectrum of FADH ${ }^{-}$(Payne and Sancar 1990) and efficiency of repair by an incident photon is lower than that of the holoenzyme because of the low extinction coefficient of $\mathrm{FADH}^{-}$.

\section{BIOCHEMICAL AND PHOTOCHEMICAL PROPERTIES OF ANIMAL CRYPTOCHROMES}

Currently, it is known that Insect Type I CRYs function as circadian photoreceptors and that Insect Type II CRYs, like the evolutionarily related vertebrate CRYs, function as repressors of Clock/Cycle (CLOCK/BMAL in vertebrates) (Zhu et al. 2005; Yuan et al. 2007). Mechanistic details of both the photoreceptor and the repressor functions of CRYs are poorly understood; it is also unknown whether the two functions are mutually exclusive.

Below, some of the physical and biochemical properties of animal CRYs are discussed. Reference will be made to plant CRYs only when it is necessary to explain a particular property of animal CRYs.

\section{Physical Properties}

Spectroscopic properties. No native CRY has been purified to date due to their low abundance; instead, the CRYs have been expressed as recombinant proteins using bacterial, insect, or mammalian cells. CRYs purified in this manner contain little to no MTHF chromophore (Lin et al. 1995; Malhotra et al. 1995; Hsu et al. 1996; Song et al. 2007). With respect to flavin content, CRYs fall into two groups. The first group, which includes Arabidopsis CRYs and Insect CRY1s can be purified from bacterial and insect expression systems with essentially stoichiometric amounts of FAD (Lin et al. 1995; Malhotra et al. 1995; Bouly et al. 2003; Özgür and Sancar 2006; Song et al. 2007). In contrast, the second group, which includes vertebrate CRYs and Insect CRY2s, contains 1-2\% FAD and trace amounts of MTHF when purified from bacterial (Hsu et al. 1996), insect (Özgür and Sancar 2006; Song et al. 2007), or mammalian (Özgür and Sancar 2003) expression systems. These findings have raised the legitimate question of whether these CRYs are in fact flavoproteins or whether they have only retained flavin-binding capacity as a functionally irrelevant evolutionary relic. To date, no photobiological activity has been associated with this group of CRYs; therefore, it is logical to conclude either that flavin binding by this group of CRYs is not relevant to their activities or that FAD has only a structural, and not catalytic, role. However, it must be noted that many photolyases, including the (6-4) photolyase of Drosophila, contain 1-5\% FAD (Zhao and Sancar 1997) when expressed in a heterologous system, yet enzymatic activities of these enzymes are absolutely dependent on this cofactor. In light of all these considerations, we believe all CRYs contain FAD as a functional cofactor. Further work is needed to test this prediction.

A second question with respect to the chromophore/ cofactor issue of CRYs is the redox status of FAD in the native CRY. Photolyases are known to utilize two-electron reduced deprotonated flavin $\left(\mathrm{FADH}^{-}\right)$as the native cofactor (Sancar 2003; Selby and Sancar 2006); however, when purified under aerobic conditions, they may have the flavin in any of the three oxidation states, $\mathrm{FADH}^{-}$, $\mathrm{FADH}^{\circ}$ (blue neutral radical), and $\mathrm{FAD}_{\text {ox }}$ (Sancar 2003). Hence, it is not possible to ascertain the redox status of the cofactor in vivo by inspecting the redox status of the flavin cofactor of purified photolyase. So far, all of the plant and Insect Type I CRYs that have been purified contain flavin in the two-electron oxidized form, $\mathrm{FAD}_{\mathrm{ox}}$ (Fig. 6). As argued for photolyases, this does not necessarily mean that the native pigment contains oxidized flavin. The resolution of this issue awaits the establishment of the CRY photocycle in an in vitro system.

Quarternary structure. All nonvisual photoreceptors that have been analyzed to date including phytochrome, phototropin, and BLUF proteins are homodimers (Christie 2007). It has been reported that AtCRY1 and AtCRY2 form homodimers and heterodimers that are essential for their in vivo function (Sang et al. 2005; Yu et al. 2007). In contrast, it was recently reported that Drosophila CRY is a monomer (Berndt et al. 2007), whereas coimmunoprecipitation experiments with recombinant mammalian CRYs suggest that they form homoand heterodimers (Partch 2006). Further work with native mammalian CRYs is needed to resolve the issue of the quaternary structure of CRYs.

\section{Biochemical Properties}

Animal CRYs exhibit a number of macromolecular interactions and enzymatic activities that may be related to their light-independent signaling. The significance of some of these interactions are well-understood, whereas others require further investigation.

DNA binding. In contrast to the crystal structure of AtCRY1 (Brautigam et al. 2004), a computational model of human CRY2 revealed that the DNA-binding groove of photolyase is conserved in human CRYs (Özgür and Sancar 2003). Not surprisingly, human CRY2 bound weakly to double-stranded DNA and with higher affinity to single-stranded DNA nonspecifically (Fig. 7A), with a $K_{\mathrm{D}} \sim 5 \times 10^{-9}$, and with slightly higher affinity to a single- 


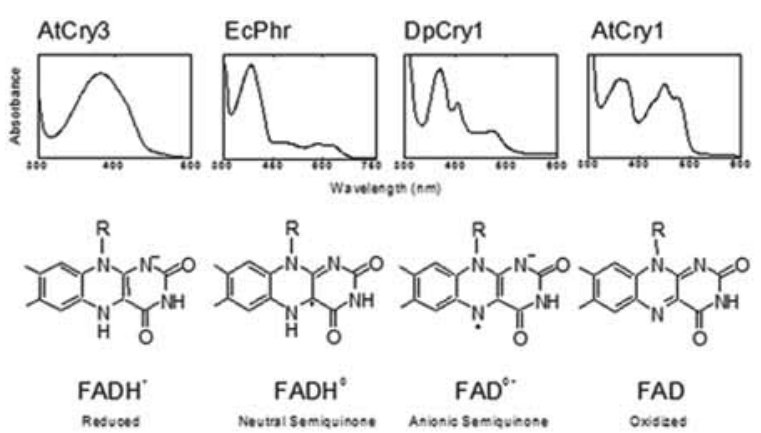

Figure 6. Spectroscopic and chemical properties of the FAD cofactor in representative members of the photolyase/CRY family. When the enzymes are purified, they may contain one or both chromophores, and the flavin cofactor may be in any of the three oxidation states (that do not necessarily represent the in vivo form of the cofactor) depending on the particular enzyme or the purification conditions. "AtCRY3" is the single-stranded DNA photolyase from Arabidopsis, which was misclassified as a CRY before discovery of its single-stranded DNA-specific photolyase activity. It contains stoichiometric MTHF and flavin in the twoelectron-reduced and deprotonated $\left(\mathrm{FADH}^{-}\right)$form. $\mathrm{EcPhr}$ is the E. coli photolyase. Even though this enzyme contains $\mathrm{FADH}^{-}$in its native state, the flavin is oxidized to the blue neutral radical (semiquinone) state when purified. The absorption spectrum shows the contribution of MTHF at $380 \mathrm{~nm}$ and of FADH ${ }^{\circ}$ in the 450-700-nm range. DpCRY1 shows the absorption spectrum of the monarch butterfly CRY1 after light exposure. When the enzyme is purified in the dark, it exhibits a $\mathrm{FAD}_{\mathrm{ox}}$ absorption spectrum. The enzyme is extremely sensitive to light, and upon light exposure, it is reduced to the flavin anion radical $\mathrm{FAD}^{-\circ}$, which is thought to be the active form of this CRYI (Song et al. 2007). AtCRY 1 shows the absorption spectrum of Arabidopsis CRY1 purified from an insect cell expression system, exhibiting the characteristic $\mathrm{FAD}_{\mathrm{ox}}$ absorption spectrum. It is unclear whether this is the physiologically relevant form of the cofactor. Note that DpCRY1 and AtCRY1 contain only trace amounts of MTHF, which does not significantly contribute to the absorption spectrum.

stranded DNA oligomer containing a (6-4) photoproduct, but there was no photorepair of the lesion. Human CRY1 exhibited similar DNA-binding properties (Özgür and Sancar 2003). Currently, the significance of these findings is not known.

Protein-protein interactions. In the Drosophila circadian clock, CRY binds to Timeless (Tim) in a light-dependent manner (Ceriani et al. 1999) and promotes ubiquitylation of Tim by Jetlag SCF E3 ligase (Koh et al. 2006), and this leads to degradation. This reaction is important to phase setting. Mammalian CRYs interact with the integral clock proteins PER, CLOCK, and BMAL1 independently of light (Griffin et al. 1999) and repress the transcriptional activity of the CLOCK/ BMAL1 complex (Kume et al. 1999; Vitaterna et al. 1999) by an ill-defined mechanism. It should be noted that even though the binding of DmCRY to DmTim in a yeast two-hybrid assay is light-dependent and that of hCRY1 and hCRY2 to hPER and hCLOCK is light-independent, this should not be taken as evidence that mammalian CRYs have no lightdependent activity. As noted above, ectopically expressed mammalian CRYs contain essentially no flavin and therefore cannot be expected to be sensitive to light.
In addition to these key interactions that make up the negative arm of the core molecular clock, human CRYs interact with several other proteins that are involved in the clock and cell cycle checkpoints. Both human CRY1 and CRY2 bind to phosphoprotein phosphatase 5 (PP5) (Zhao and Sancar 1997) and inhibit PP5 activity toward autophosphorylated CKIE and in so doing inhibit phosphorylation and subsequent degradation of PER1 and PER2 (Partch et al. 2006). As a consequence, the CRY-PP5 interaction has a role in consolidating the core molecular clock. Similarly, FBXL3 E3 ubiquitin ligase binds to CRY1 and CRY2 and ubiquitylates them, leading to their timely degradation. In the absence of FBXL3, CRYs accumulate, leading to exaggerated inhibition of CLOCK/BMAL1 and abnormal circadian period and phase response (Busino et al. 2007; Godinho et al. 2007; Siepka et al. 2007). Finally, human CRYs interact with the Tim checkpoint/circadian protein and in so doing couple the circadian cycle to the cell cycle (Ünsal-Kaçmaz et al. 2005).

In Arabidopsis, both CRY1 and CRY2 interact directly with the E3 ubiquitin ligase COP1 in a light-independent manner through their carboxy-terminal domains (Wang et al 2001; Yang et al 2001). In the dark, the CRY-COP1 complex is active as an E3 enzyme, ubiquitylating transcription factors, such as HY5, and thus inhibiting transcription. When exposed to blue light, the CRY-COP1 complex does not dissociate, but apparently a lightinduced conformational change in the carboxyl termini of CRY results in inhibition of COP1 activity and thus leads to transcriptional induction of blue-light-responsive

A

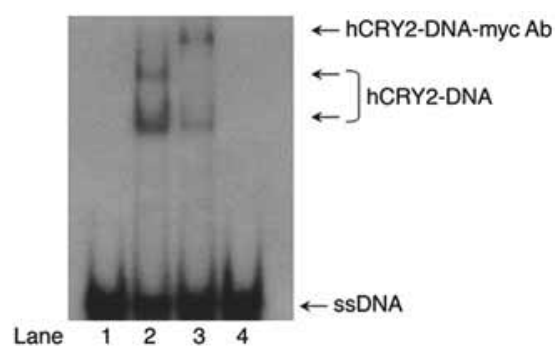

B

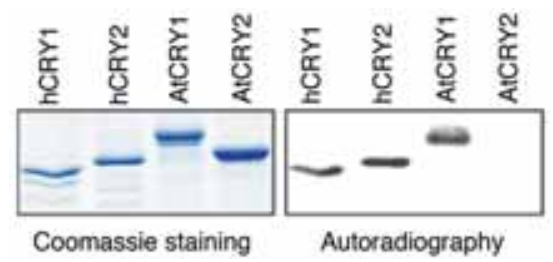

Figure 7. DNA binding and kinase activities of CRYs. $(A)$ Electrophoretic mobility-shift assay showing binding of hCRY2 to single-stranded DNA. The DNA-protein complex is supershifted by antibodies to an epitope tag on human CRY2. The radiolabeled single-stranded DNA was incubated with myctagged human CRY2 (lanes 2 and 3) or anti-myc antibodies (lanes 3 and 4 ) before loading on the gel. (B) Kinase activities of Arabidopsis and human CRYs in vitro. Purified recombinant CRYs were incubated with $\left[\gamma^{32} \mathrm{P}\right] \mathrm{ATP}$, separated on an SDSPAGE gel, and then analyzed by Coomassie Blue staining (left) or autoradiography (right). (Reprinted, with permission, from Özgür and Sancar [2003] and Özgür and Sancar [2006] [both (C) American Chemical Society].) 
genes in Arabidopsis that are involved in growth and differentiation (Yang et al 2000; Wang et al. 2001). It is thought that the CRY-COP1 interaction is unique to plant CRYs (Yang et al. 2001); however, more work is needed to find out whether or not mammalian CRYs bind COP1 and exert some control over protein degradation.

ATP binding and autokinase activity. Autophosphorylation is a common property of all photosensory pigments including phytochromes and phototropins (Christie 2007). However, it also appears that the autokinase activity is dispensable for both phytochrome (Matsushita et al. 2003) and phototropin (Kagawa et al. 2004) functions. It was found that both AtCRY1 and AtCRY2 are phosphorylated in vivo upon blue light exposure (Shalitin et al. 2002; 2003). Later, it was shown that purified AtCRY1 bound ATP stoichiometrically and that both AtCRY1 (Bouly et al. 2003; Shalitin et al. 2003) and human CRY1 (Bouly et al. 2003) were autophosphorylated in a FAD- and blue-light-dependent manner. In line with these biochemical findings, the crystal structure of the AtCRY1 PHR domain contained an ATP analog in the cavity leading to FAD where the pyrimidine dimer binds in photolyase (Brautigam et al. 2004). It is unclear, however, how this ATP could be used for autophosphorylation because there are no serine/threonine residues within reasonable distance of the binding site for chemical attack on ATP to achieve phosphorylation. However, the crystal structure is missing the carboxy-terminal domain, and hence, it is conceivable that a residue in the missing domain participates in the kinase activity.

Experiments with human CRYs cast some doubt about the role of the weak autokinase activity of CRYs in their function (Özgür and Sancar 2006). First, it was found that no correlation exists between the presence of FAD and the autokinase activity. The human CRY1 and CRY2 purified from baculovirus/insect cell system contained either no or only trace flavin, yet autophosphorylated to the same level as AtCRY1, which contained stoichiometric flavin (Fig. 7B). Second, purified AtCRY2 which is known to be phosphorylated in vivo in response to blue light was not phosphorylated in the dark or under blue light. Finally, the kinetics and extent of AtCRY1 autophosphorylation were essentially identical under blue light and in the dark under our conditions. The cause of these contradictory results is not known, and further work is needed to understand the significance of ATP binding to CRYs and the role, if any, of autokinase activity in CRY function.

\section{The "Trp Triad" and Photoreduction}

Excitation of flavin by blue light in the majority of flavoproteins leads to quenching of the excited state at a rate much faster than that of free flavin (see Sancar 2003). This is, in general, due to electron transfer from redoxactive amino acids such as tryptophan, tyrosine, and histidine to the excited-singlet-state flavin that is a potent oxidant. This property of flavoproteins has been extensively used to study the physicochemical aspects of intraprotein electron transfer (Zhong 2007). Naturally, in enzymes that utilize ground-state flavin to catalyze redox reactions such as glucose oxidase, which carries out catalysis independently of light, the photoinduced reduction of flavin has no bearing on enzyme activity under physiological conditions. The significance of photoinduced electron transfer from aromatic amino acids to the flavin photoreceptor is more difficult to ascertain. Stringent criteria must be applied to determine whether photoreduction of the flavin is a side reaction with no biological relevance or a key step in the photocycle of these flavinbased photoreceptors. In E. coli photolyase, the "Trp triad," FADH ${ }^{\circ} \leftarrow$ Trp- $382 \leftarrow$ Trp- $359 \leftarrow$ Trp-306 is the predominant photoreduction pathway with Trp-306 being the ultimate electron donor (Fig. 8A) (Li et al. 1991; Park et al. 1995; Kavakli and Sancar 2004). Interestingly, the residues of this "Trp triad" are conserved in most DNA photolyases and CRYs (Kim et al. 1993; Lin and Shalitin 2003; Partch and Sancar 2005; Zeugner et al. 2005). It has been unequivocally shown that this pathway has no role in the photocycle of photolyase (Li et al. 1991; Kavaklı and Sancar 2004). In contrast, as will be discussed below, some studies have suggested that photoinduced electron

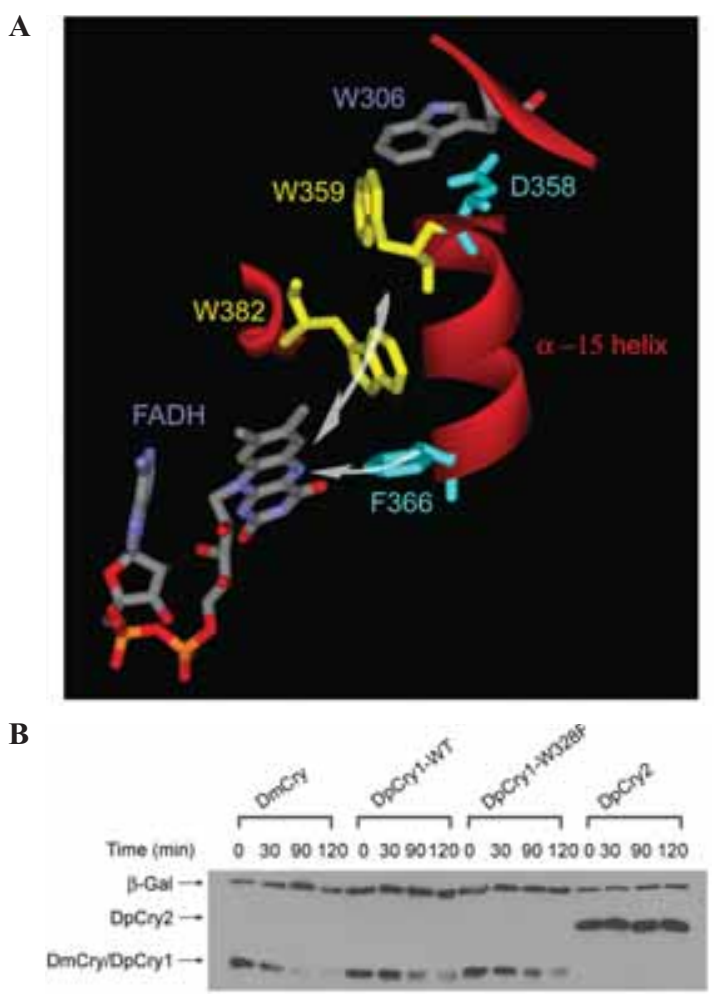

Figure 8. Trp triad and CRY function. (A) The "Trp triad" in $E$. coli photolyase. Excitation of FADH ${ }^{\circ}$ by light leads to its photoreduction by electron transfer from Trp306 through either electron hopping (W382 $\leftarrow \mathrm{W} 359 \leftarrow \mathrm{W} 306)$ or electron tunneling (F366 $\leftarrow \alpha 15$-helix $\leftarrow$ W306). Mutating "Trp-306 to Phe-306 blocks both pathways. $(B)$ Effect of blocking electron transfer to $\mathrm{FAD}_{\mathrm{ox}}$ in $\mathrm{DpCryl}$ on its photoreception activity. Blocking the intraprotein electron transfer by the W328F mutation (the equivalent of E. coli Phr W306) has no effect on the photoreception activity of DpCRY1 as measured by its photoinduced degradation in $\mathrm{S} 2$ cells. ( $A$, Reprinted, with permission, from Saxena et al. 2004 [(c) American Chemical Society]; B, reprinted, with permission, from Song et al. 2007 [C ASBMB].) 
transfer is the key photophysical event in the CRY photocycle (Zeugner et al. 2005; Banerjee et al. 2007; Bouly et al. 2007).

\section{FUNCTIONAL PROPERTIES OF ANIMAL CRYPTOCHROME}

\section{Cryptochrome Photocycle}

The photocycle of a photosensory pigment is the sequence of physicochemical transitions that take place from the time of absorption of a photon to generation of a transient signaling state, followed by deactivation of the pigment to the ground state. Of the flavin-based photoreceptors, the photocycles of photolyase and phototropin are well-characterized. The former involves a photoinduced cyclic electron transfer (Sancar 2003) and the latter involves a photoinduced covalent bond formation between the protein and $\mathrm{FAD}$, followed by a conformational change (Swartz et al. 2001; Harper et al. 2003). Therefore, it is tempting to consider the CRY photocycle in terms of one of these two general models. Because of the phylogenetic and structural relations, one is further tempted to think that the "photolyase model" is more likely to be applicable to CRYs. However, two observations indicate that the photolyase model cannot apply to CRY in the strict sense. First, photolyase binds its substrate in a light-independent reaction and then catalysis is initiated by light, whereas DmCRY binds to Tim only after excitation of CRY by light (Ceriani et al. 1999). Second, photolyase can undergo hundreds of photocycles without being degraded whereas all CRYs known to function as photoreceptors, with the exception of AtCRY1, undergo photoinduced degradation (Lin et al. 1998; Yuan et al. 2007).

In addition to the evidence against the "photolyase model" for the CRY photocycle, a number of reports appear to support a "phototropin-like model" involving photoinduced conformational change. Most of these studies have been done on Arabidopsis CRYs. First, it has been reported that AtCRY1 and AtCRY2 made in insect cells contain flavin in the $\mathrm{FAD}_{\mathrm{ox}}$ state and that exposure of the insect cells expressing the CRYs to blue light reduces the $\mathrm{FAD}_{\mathrm{ox}}$ to a $\mathrm{FADH}^{\circ}$ neutral radical (Banerjee et al. 2007; Bouly et al. 2007). This was complemented by in vitro experiments that demonstrated that exposure of AtCRYs to light followed by a dark phase under aerobic conditions cause the following transitions:

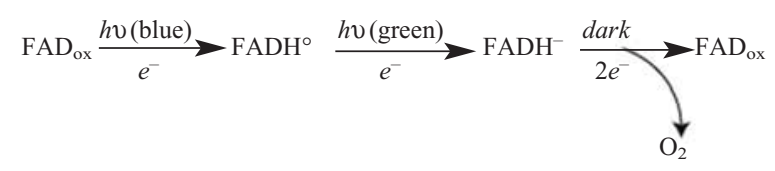

This led to the conclusion that the $\mathrm{CRY}\left(\mathrm{FADH}^{\circ}\right)$ was the signaling state of Arabidopsis CRYs and that further exposure of the plant to green light where $\mathrm{FADH}^{\circ}$ absorbs turned off the signal by reducing the radical to $\mathrm{FADH}^{-}$. Conversely, dark incubation of $\mathrm{CRY}\left(\mathrm{FADH}^{-}\right)$resulted in reoxidization back to the photoactive $\mathrm{FAD}_{\mathrm{ox}}$ form. Second, it was reported that blue light activated the AtCRY1 kinase (Bouly et al. 2003) and that this activa- tion was dependent on photoinduced electron transfer through the so-called "Trp triad." Mutations of one of the three tryptophans to phenylalanine blocked photoreduction and abolished light-induced kinase activity (Zeugner et al. 2005). Finally, it was shown by two independent methods that blue light causes significant conformational change in the carboxy-terminal domain of AtCRY1, which is critical for signaling in vivo (Partch et al. 2005; Kottke et al. 2006).

All of these observations are, however, subject to some serious caveats. First, any overproduced flavoprotein may be subject to photoreduction in the reducing milieu of insect cells, and hence, photoreduction of an overproduced protein need not necessarily be on the signaling pathway. Second, reports that blue light stimulates AtCRY1 kinase activity have not been reproducible in a recent exhaustive in vitro study (Özgür and Sancar 2006). Similarly, the report that blocking photoreduction by mutating the "Trp triad" abolishes the blue-light-induced kinase activity has a serious drawback: The mutant proteins had only $20 \%$ basal kinase (in the dark) activity compared to the wild-type enzyme (Zeugner et al. 2005), suggesting that the mutations must have caused partial misfolding of the proteins and making it difficult to interpret the lack of light stimulation, which again has not been reproducible. Third, this model predicts that the action spectrum for hypocotyl growth inhibition would be very similar to the absorption spectrum of $\mathrm{FAD}_{\mathrm{ox}}$. However, the actual action spectrum of hypocotyl growth inhibition in Arabidopsis is quite flat in the 390-480-nm range (Ahmad et al. 2002). Finally, DmCry missing the carboxy-terminal extension is capable of photoreception and phototransduction, suggesting that at least in some CRYs, the carboxy-terminal domain is not necessary for photosignaling (Busza et al. 2004).

More recently, it was reported that the photoreduction of the flavin in Insect CRY1 to anion radical (Denaro 2006; Berndt et al. 2007) and its reversal in the dark to $\mathrm{FAD}_{\mathrm{ox}}$ constituted the photocycle of this class of CRYs (Berndt et al. 2007):

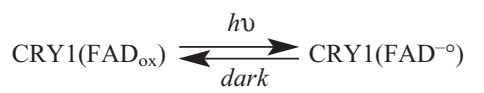

However, the action spectrum of DmCRY does not match the absorption spectrum of $\mathrm{FAD}_{\text {ox }}$ (Van Vickle-Chavez and Van Gelder 2006), and the first experimental test of this model with the photosensitive monarch butterfly CRY, DpCRY1, did not support the model (Fig. 8B): Mutating the ultimate electron donor of the "Trp triad" blocked photoreduction of $\mathrm{FAD}_{\mathrm{ox}}$ to $\mathrm{FAD}^{\circ-}$ in vitro. However, the mutation had no effect in vivo on the photoreceptor activity of DpCry1 as revealed by blue-lightinduced degradation of the CRY (Song et al. 2007). In light of this finding and the recently published action spectrum of DmCRY (a member of type 1 CRYs) that does not match the absorption spectrum of $\mathrm{FAD}_{\text {ox }}$ (VanVickle-Chavez and Van Gelder 2007), we conclude that most likely, $\mathrm{FAD}_{\text {ox }}-h v \rightarrow \mathrm{FAD}^{-\circ}$ is not part of the insect CRY photocycle and that the active form of type I CRYs may actually contain $\mathrm{FAD}^{-\circ}$. 


\section{Role of Cryptochrome in Magnetoreception}

How organisms sense the earth's magnetic field to guide activities such as migration is not known. CRYs are currently considered candidate "magnetoreceptors" functioning as sensors via a postulated mechanism, "chemical magnetoreception," that has been gaining popularity (Ritz et al. 2000; Johnsen and Lohmann 2005). Theoretically, the weak magnetic field of the earth could influence the outcome of certain biochemical reactions by influencing the correlation of spin states of radical pair intermediates, such as those generated in photoactivated states of photolyases and possibly CRYs. This mechanism raises the possibility that CRYs have a substrate upon which they act and that the nature of the product or the rate of the photochemical reaction is influenced by the physical orientation of the CRY with respect to the magnetic field. For magnetic field lines to be sensed, this hypothesis requires that the CRYs be fixed in a uniform position within the architecture of the photoreceptive cells and tissue, for example, in unidirectional layers. In this way, all of the CRYs will be influenced uniformally by the magnetic field. Consequently, the rate of CRY signaling will vary as the position of the organism changes. Several aspects of migratory organisms are consistent with this mechanism. Light and eyes are required for certain migratory behaviors, and CRY has been found in photoreceptor tissues of several organisms. In the migratory bird garden warbler (Sylvia borin), CRY expression was localized in a subset of retinal ganglion cells associated with magnetic orientation behavior (Mouritsen et al. 2004). Other theories and potential magnetoreceptors exist; however, this is clearly a fertile field of research that will possibly include characterization of exciting new CRY functions as it develops.

\section{Role of Cryptochrome in Cell Cycle Regulation}

The circadian cycle and cell cycle are two global regulatory mechanisms that affect nearly all aspects of cellular physiology; therefore, it is to be expected that these two regulatory pathways might exhibit some overlap. In organisms ranging from Chlamydomonas (Nikaido and Johnson 2000) to zebra fish (Dekens et al. 2003) to humans (Bjarnason and Jordan 2000), the circadian rhythm affects the phasing of the cell cycle. Inversely, the cell cycle also gates the phase of the circadian cycle (Nagoshi et al. 2004) as a further indicator of the intimate connection between the two pathways. CRY affects the mammalian cell cycle checkpoints by two mechanisms. First, it interacts with the Timeless protein which, in turn, interacts with the damage sensor ATR and the signal transducer Chk1 kinase and in so doing, CRY directly participates in the DNA-damage checkpoint (ÜnsalKaçmaz et al. 2005). Second, CRYs in their capacities as negative regulators of clock-controlled genes repress the expression of Weel mitotic kinase, and as a consequence, in $C r y 1^{-1-}$;Cry2 $2^{-1-}$ cells, Weel is elevated (Matsuo et al. 2003; Gauger and Sancar 2005). However, the effect of Wee1 elevation on cell cycle checkpoint may or may not be apparent depending on the cell type (Matsuo et al. 2003; Gauger and Sancar 2005); in fact, it was found that Cry $1^{-1-} ;$ Cry $2^{-/-}$mice were not measurably different from the wild-type mice in terms of chronic effects of ionizing radiation (Gauger and Sancar 2005) and were more resistant to the acute genotoxic effects of cyclophosphamide (Gorbacheva et al. 2005; Kondratov et al. 2007). Finally, CRY regulates PP5 activity, which in turn regulates ATM activity (Partch et al. 2006; Yong et al. 2007), and through this pathway, CRY is expected to have a role in cellular response to ionizing radiation which is controlled to a large extent by ATM (Sancar et al. 2004). Clearly, the CRY-cell cycle checkpoint connection in particular and circadian cycle-cell cycle connection in general are fertile fields for further research and biomedical exploitation (Kondratov et al. 2007; Hunt and Sassone-Corsi 2007).

\section{CONCLUSION}

At present, the photochemical reaction initiating CRY signal transduction is not known. Two general models that might be referred to as the "photolyase model" (photoinduced cyclic electron transfer) and the "phototropin model" (photoinduced conformational change) have been proposed for the photocycle (Fig. 9). There are experimental data for and against each of these models, and further work is required to find out which of these models, if any, apply to the CRY photocycle. In addition to this
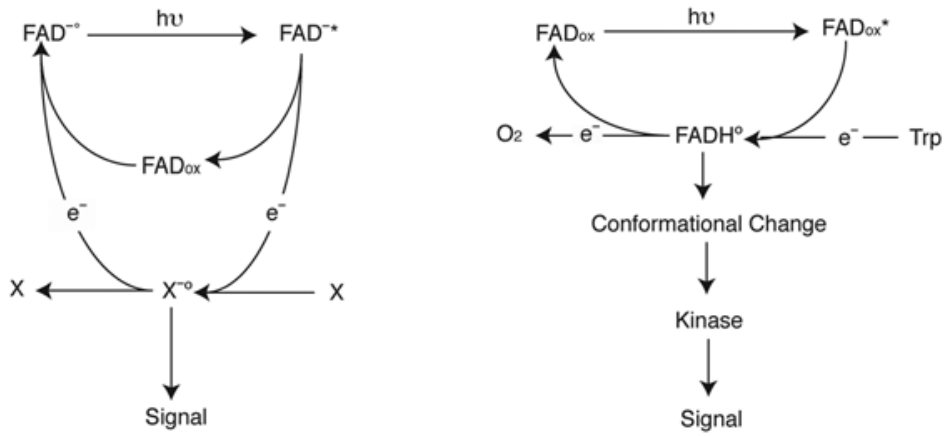

Figure 9. Two models for the CRY cycle. In the "Photolyase Model," photoinduced cyclic electron transfer from reduced or semireduced (shown) flavin to an unknown substrate generates a radical that initiates the signal; back electron transfer turns off the light signal (Song et al. 2007). In the "Phototropin Model," light-induced electron transfer from a Trp residue in the apoenzyme to $\mathrm{FAD}_{\text {ox }}$ generates a flavin radical concomitant with significant conformational change in the CRY that activates the CRY's autokinase activity and affects CRY's interactions with downstream proteins, such as COP1, modulating their activity to transmit signal (Bouly et al. 2007). 


\section{STRUCTURE AND FUNCTION OF ANIMAL CRYPTOCHROMES}

issue, the following questions remain to be addressed for a more comprehensive understanding of animal CRY structure and function: (1) Do Insect CRY1s act as transcriptional repressors? (2) Do vertebrate CRYs and Insect CRY2s function as photoreceptors? (3) Do vertebrate CRYs and Insect CRY2s contain FAD and if so what is the function of FAD? (4) Do CRYs from plant and animal sources have a second chromophore? (5) What is the role of CRY kinase activity in signaling?

\section{ACKNOWLEDGMENTS}

This work was supported by National Institutes of Health grant GM31082. This review is in large part based on papers by Özgür and Sancar (2003, 2006), Sancar (2004), Partch (2006), and Song et al. (2007).

\section{REFERENCES}

Adams M.D., Kerlavage A.R., Fleischmann R.D., Fuldner R.A., Bult C.J., Lee N.H., Kirkness E.F., Weinstock K.G., Gocayne J.D., and White O., et al. 1995. Initial assessment of human gene diversity and expression patterns based upon 83 million nucleotides of cDNA sequence. Nature 377: 3.

Ahmad M. and Cashmore A.R. 1993. HY4 gene of A. thaliana encodes a protein with characteristics of a blue-light photoreceptor. Nature 366: 162.

Ahmad M., Grancher N., Heil M., Black R.C., Giovani B., Galland P., and Lardemer D. 2002. Action spectrum for cryptochrome-dependent hypocotyl growth inhibition in Arabidopsis. Plant Physiol. 129: 774.

Banerjee R. and Batschauer A. 2005. Plant blue-light receptors. Planta 220: 498.

Banerjee R., Schleicher E., Meier S., Munoz Viana R., Pokorny R., Ahmad M., Bittl R., and Batschauer A. 2007. The signaling state of Arabidopsis cryptochrome 2 contains flavin semiquinone. J. Biol. Chem. 282: 14916.

Berndt A., Kottke T., Breitkreuz H., Dvorsky R., Hennig S., Alexander M., and Wolf E. 2007. A novel photoreaction mechanism for the circadian blue light photoreceptor Drosophila cryptochrome. J. Biol. Chem. 282: 13011.

Bjarnason G.A. and Jordan R. 2000. Circadian variation of cell proliferation and cell cycle protein expression in man: Clinical implications. Prog. Cell Cycle Res. 4: 193.

Bouly J.P., Giovani B., Djamei A., Mueller M., Zeugner A., Dudkin E.A., Batschauer A., and Ahmad M. 2003. Novel ATP-binding and autophosphorylation activity associated with Arabidopsis and human cryptochrome-1. Eur. J. Biochem. 270: 2921.

Bouly J.P., Schleicher E., Dionisio-Sese M., Vandenbussche F., Van Der Straeten D., Bakrim N., Meier S., Batschauer A., Galland P., Bittl R., and Ahmad M. 2007. Cryptochrome blue light photoreceptors are activated through interconversion of flavin redox states. J. Biol. Chem. 282: 9383.

Brautigam C.A., Smith B.S., Ma Z., Palnitkar M., Tomchick D.R., Machius M., and Deisenhofer J. 2004. Structure of the photolyase-like domain of cryptochrome 1 from Arabidopsis thaliana. Proc. Natl. Acad. Sci. 101: 12142.

Busino L., Bassermann F., Maiolica A., Lee C., Nolan P.M. Godinho S.I., Draetta G.F., and Pagano M. 2007. SCF Fbx13 controls the oscillation of the circadian clock by directing the degradation of cryptochrome proteins. Science 316: 900.

Busza A., Emery-Le M., Rosbash M., and Emery P. 2004. Roles of the two Drosophila CRYPTOCHROME structural domains in circadian photoreception. Science 304: 1503.

Cashmore A.R. 2003. Cryptochromes: Enabling plants and animals to determine circadian time. Cell 114: 537.

Ceriani M.F., Darlington T.K., Staknis D., Mas P., Petti A.A., Weitz C.J., and Kay S.A. 1999. Light-dependent sequestration of TIMELESS by CRYPTOCHROME. Science 285: 553.
Christie J.M. 2007. Phototropin blue-light receptors. Annu. Rev. Plant Biol. 58: 21.

Collins B., Mazzoni E.O., Stanewsky R., and Blau J. 2006. Drosophila CRYPTOCHROME is a circadian transcriptional repressor. Curr. Biol. 16: 441.

Darwin C. 1881. The power of movement in plants. Da Capo Press, New York.

Dekens M.P., Santoriello C., Vallone D., Grassi G., Whitmore D., and Foulkes N.S. 2003. Light regulates the cell cycle in zebrafish. Curr. Biol. 13: 2051.

Denaro T.R. 2006. "Purification and characterization of the Danaus plexippus cryptochromes." M.S. thesis, University of North Carolina, Chapel Hill.

Emery P., So W.V., Kaneko M., Hall J.C., and Rosbash M. 1998. CRY, a Drosophila clock and light-regulated cryptochrome, is a major contributor to circadain rhythm resetting and photosensitivity. Cell 95: 669.

Fujihashi M., Numoto N., Kobayashi Y., Mizushima A., Tsujimura M., Nakamura A., Kawarabayasi Y., and Miki K. 2007. Crystal structure of archaeal photolyase from Sulfolobus tokodaii with two FAD molecules: Implication of a novel light-harvesting cofactor. J. Mol. Biol. 365: 903.

Gauger M.A. and Sancar A. 2005. Cryptochrome, circadian cycle, cell cycle checkpoints, and cancer. Cancer Res. 65: 6828 .

Gehring W. and Rosbash M. 2003. The coevolution of blue-light photoreception and circadian rhythms. J. Mol. Evol. 57: S286.

Gekakis N., Staknis D., Nguyen H.B., Davis F.C., Wilsbacher L.D., King D.P., Takahashi J.S., and Weitz C.J. 1998. Role of CLOCK protein in the mammalian circadian mechanism. Science 280: 1564.

Godinho S.I., Maywood E.S., Shaw L., Tucci V., Barnard A.R., Busino L., Pagano M., Kendall R., Quawailid M.M., Romero M.R., O'neill J., Chesham J.E., Brooker D., Lalanne Z., Hastings M.H., and Nolan P.M. 2007. The after-hours mutant reveals a role for Fbxl3 in determining mammalian circadian period. Science 316: 897.

Gorbacheva V.Y., Kondratov R.V., Zhang R., Cherukuri S., Gudkov A.V., Takahashi J.S., and Antoch M.P. 2005. Circadian sensitivity to the chemotherapeutic agent cyclophosphamide depends on the funtional status of the CLOCK/BMAL1 transactivation complex. Proc. Natl. Acad. Sci. 102: 3407.

Gressel J. 1977. Blue light photoreceptors. Photochem. Photobiol. 30: 749.

Griffin E.A., Jr., Staknis D., and Weitz C.J. 1999. Light-independent role of CRY1 and CRY2 in the mammalian circadian clock. Science 286: 768.

Guo H., Yang H., Mockler T.C., and Lin C. 1998. Regulation of flowering time by Arabidopsis photoreceptors. Science 279: 1360 .

Harper S.M., Neil L.C., and Gardner K.H. 2003. Structural basis of a phototropin light switch. Science 301: 1541.

Hsu D.S., Zhao X., Zhao S., Kazantsev A., Wang R.P., Todo T., Wei Y.F., and Sancar A. 1996. Putative human blue-light photoreceptors hCRY1 and hCRY2 are flavoproteins. Biochemistry 35: 13871.

Huang Y., Baxter R., Smith B.S., Partch C.L., Colbert C.L., and Deisenhofer J. 2006. Crystal structure of cryptochrome 3 from Arabidopsis thaliana and its implications for photolyase activity. Proc. Natl. Acad. Sci. 103: 17701.

Hunt T. and Sassone-Corsi P. 2007. Riding tandem: Circadian clocks and the cell cycle. Cell 129: 461.

Johnsen S. and Lohmann K.J. 2005. The physics and neurobiology of magnetoreception. Nat. Rev. Neurosci. 6: 703.

Johnson J.L., Hamm-Alvarez S., Payne G., Sancar G.B., Rajagopalan K.V., and Sancar A. 1988. Identification of the second chromophore of Escherichia coli and yeast DNA photolyases as 5,10-methenyltetrahydrofolate. Proc. Natl. Acad. Sci. 85: 2046.

Kagawa T., Kasahara M., Abe T., Yoshida S., and Wada M. 2004. Function analysis of phototropin2 using fern mutants deficient in blue light-induced chloroplast avoidance movement. Plant Cell Physiol. 45: 416. 
Kao Y.T., Saxena C., Wang L., Sancar A., and Zhong D. 2005. Direct observation of thymine dimer repair in DNA by photolyase. Proc. Natl. Acad. Sci. 102: 16128.

Kavakli I.H. and Sancar A. 2004. Analysis of the role of intraprotein electron transfer in photoreactivation by DNA photolyase in vivo. Biochemistry 43: 15103.

Kim S.T., Malhotra K., Taylor J.S., and Sancar A. 1996. Purification and partial characterization of (6-4) photoproduct DNA photolyase from Xenopus laevis. Photochem. Photobiol. 63: 292.

Kim S.T., Sancar A., Essenmacher C., and Babcock G.T. 1993. Time-resolved EPR studies with DNA photolyase: Excitedstate $\mathrm{FADH}^{\circ}$ abstracts an electron from Trp-306 to generate $\mathrm{FADH}^{-}$, the catalytically active form of the cofactor. Proc. Natl. Acad. Sci. 90: 8023.

Koh K., Zheng X., and Sehgal A. 2006. JETLAG resets the Drosophila circadian clock by promoting light-induced degradation of TIMELESS. Science 312: 1809.

Kondratov R.V., Gorbacheva V.Y., and Antoch M.P. 2007. The role of mammalian circadian proteins in normal physiology and genotoxic stress responses. Curr. Top. Dev. Biol. 78: 173.

Koornneef M., Rolf E., and Spruit C.J.P. 1980. Genetic control of light-inhibited hypocotyl elongation in Arabidopsis thaliana. Z. Pflanzenphysiol. 100: 147.

Kottke T., Batschauer A., Ahmad M., and Heberle J. 2006. Bluelight-induced changes in Arabidopsis cryptochrome 1 probed by FTIR difference spectroscopy. Biochemistry 45: 2472.

Kume K., Zylka M.J., Sriram S., Shearman L.P., Weaver D.R., Jin X., Maywood E.S., Hastings M.H., and Reppert S.M. 1999. mCRY1 and mCRY2 are essential components of the negative limb of the circadian clock feedback loop. Cell 98: 193.

Li J., Uchida T., Todo T., and Kitagawa T. 2006. Similarities and differences between cyclobutane pyrimidine dimer photolyase and (6-4) photolyase as revealed by resonance Raman spectroscopy: Electron transfer from the FAD cofactor to ultraviolet-damaged DNA. J. Biol. Chem. 281: 25551.

Li Y.F., Heelis P.F., and Sancar A. 1991. Active site of DNA photolyase: Tryptophan-306 is the intrinsic hydrogen atom donor essential for flavin radical photoreduction and DNA repair in vitro. Biochemistry 30: 6322.

Li Y.F., Kim S.T., and Sancar A. 1993. Evidence for lack of DNA photoreactivating enzyme in humans. Proc. Natl. Acad. Sci. 90: 4389 .

Lin C. and Shalitin D. 2003. Cryptochrome structure and signal transduction. Annu. Rev. Plant Biol. 54: 469.

Lin C., Yang H., Guo H., Mockler T., Chen J., and Cashmore A.R. 1998. Enhancement of blue-light sensitivity of Arabidopsis seedlings by a blue light receptor cryptochrome 2. Proc. Natl. Acad. Sci. 95: 2686.

Lin C., Robertson D.E., Ahmad M., Raibekas A.A., Jorns M.S., Dutton P.L., and Cashmore A.R. 1995. Association of flavin adenine dinucleotide with the Arabidopsis blue light receptor CRY1. Science 269: 968

Losi A. 2007. Flavin-based blue-light photosensors: A photophysics update. Photochem. Photobiol. (in press).

Lowrey P.L. and Takahashi J.S. 2004. Mammalian circadian biology: Elucidating genome-wide levels of temporal organization. Annu. Rev. Genomics Hum. Genet. 5: 407.

Malhotra K., Kim S.T., Batschauer A., Dawut L., and Sancar A. 1995. Putative blue-light photoreceptors from Arabidopsis thaliana and Sinapis alba with a high degree of sequence homology to DNA photolyase contain the two photolyase cofactors but lack DNA repair activity. Biochemistry 34: 6892.

Matsuo T.S., Yamaguchi S., Mitsui S., Emi A., Shimoda F., and Okamura H. 2003. Control mechanism of the circadian clock for timing of cell division in vivo. Science 302: 255 ,

Matsushita T., Mochizuki N., and Nagatani A. 2003. Dimers of the $\mathrm{N}$-terminal domain of phytochrome $\mathrm{B}$ are functional in the nucleus. Nature 424: 571

Millar A.J. and Kay S.A. 1997. The genetics of phototransduction and circadian rhythms in Arabidopsis. Bioessays 19: 209.

Miyamoto Y. and Sancar A. 1998. Vitamin B2-based blue-light photoreceptors in the retinohypothalamic tract as the photoactive pigments for setting the circadian clock in mammals. Proc. Natl. Acad. Sci. 95: 6097.

- 1999. Circadian regulation of cryptochrome genes in the mouse. Brain Res. Mol. Brain Res. 71: 238.

Mouritsen H., Janssen-Bienhold U., Liedvogel M., Feenders G., Stalleicken J., Dirks P., and Weiler R. 2004. Cryptochromes and neuronal-activity markers colocalize in the retina of migratory birds during magnetic orientation. Proc. Natl. Acad. Sci. 101: 14294 .

Nagoshi E.C., Saini C., Bauer C., Laroche T., Naef F., and Schibler U. 2004. Circadian gene expression in individual fibroblasts: Cell-autonomous and self-sustained oscillators pass time to daughter cells. Cell 119: 693.

Nikaido S.S. and Johnson C.H. 2000. Daily and circadian variation in survival from ultraviolet radiation in Chlamydomonas reinhardtii. Photochem. Photobiol. 71: 758.

Özgür S. and Sancar A. 2003. Purification and properties of human blue-light photoreceptor cryptochrome 2 . Biochemistry 42: 2926.

. 2006. Analysis of autophosphorylating kinase activities of Arabidopsis and human cryptochromes. Biochemistry 45: 13369

Park H.W., Kim S.T., Sancar A., and Deisenhofer J. 1995. Crystal structure of DNA photolyase from Escherichia coli. Science 268: 1866.

Partch C.L. 2006. "Signal transduction mechanisms of cryptochrome." Ph.D. thesis, University of North Carolina, Chapel Hill.

Partch C.L. and Sancar A. 2005. Photochemistry and photobiology of cryptochrome blue-light photopigments: The search for a photocycle. Photochem. Photobiol. 81: 1291.

Partch C.L., Clarkson M.W., Özgür S., Lee A.L., and Sancar A. 2005. Role of structural plasticity in signal transduction by the cryptochrome blue-light photoreceptor. Biochemistry 44: 3795.

Partch C.L., Shields K.F., Thompson C.L., Selby C.P., and Sancar A. 2006. Posttranslational regulation of the mammalian circadian clock by cryptochrome and protein phosphatase 5. Proc. Natl. Acad. Sci. 103: 10467.

Payne G. and Sancar A. 1990. Absolute action spectrum of EFADH2 and E-FADH2-MTHF forms of Escherichia coli DNA photolyase. Biochemistry 29: 7715.

Pittendrigh C.S. 1993. Temporal organization: Reflections of a Darwinian clock-watcher. Annu. Rev. Physiol. 55: 16

Reppert S.M. and Weaver D.R. 2002. Coordination of circadian timing in mammals. Nature 418: 935.

Ritz T., Adem S., and Schulten K. 2000. A model for photoreceptor-based magnetoreception in birds. Biophys. J. 78: 707.

Sancar A. 2000. Cryptochrome: The second photoactive pigment in the eye and its role in circadian photoreception. Annu. Rev. Biochem. 69: 31 .

2003. Structure and function of DNA photolyase and cryptochrome blue-light photoreceptors. Chem. Rev. 103: 2203. . 2004. Regulation of the mammalian circadian clock by cryptochrome. J. Biol. Chem. 279: 34079.

Sancar A., Lindsey-Boltz L.A., Ünsal-Kaçmaz K., and Linn S. 2004. Molecular mechanisms of mammalian DNA repair and the DNA damage checkpoints. Annu. Rev. Biochem. 73: 39.

Sang Y., Li Q.H., Rubio V., Zhang Y.C., Mao J., Deng X.W., and Yang H.Q. 2005. N-terminal domain-mediated homodimerization is required for photoreceptor activity of Arabidopsis CRYPTOCHROME 1. Plant Cell 17: 1569.

Saxena C., Sancar A., and Zhong D. 2004. Femtosecond dynamics of DNA photolyase: Energy transfer of antenna initiation and electron transfer of cofactor reduction. J. Phys. Chem. B 108: 18026.

Selby C.P. and Sancar A. 2006. A cryptochrome/photolyase class of enzymes with single-stranded DNA-specific photolyase activity. Proc. Natl. Acad. Sci. 103: 17696.

Selby C.P., Thompson C., Schmitz T.M., Van Gelder R.N., and Sancar A. 2000. Functional redundancy of cryptochromes and classical photoreceptors for nonvisual ocular photoreception in mice. Proc. Natl. Acad. Sci. 97: 14697. 


\section{STRUCTURE AND FUNCTION OF ANIMAL CRYPTOCHROMES}

Shalitin D., Yu X., Maymon M., Mockler T., and Lin C. 2003. Blue light-dependent in vivo and in vitro phosphorylation of Arabidopsis cryptochrome 1. Plant Cell 15: 2421

Shalitin D., Yang H., Mockler T.C., Maymon M., Guo H., Whitelam G.C., and Lin C. 2002. Regulation of Arabidopsis cryptochrome 2 by blue-light-dependent phosphorylation. Nature 417: 763

Siepka S.M., Yoo S.H., Park J., Song W., Kumar V., Hu Y., Lee C., and Takahashi J.S. 2007. Circadian mutant overtime reveals F-box protein FBXL3 regulation of cryptochrome and period gene expression. Cell 129: 1011

Somers D.E., Devlin P.F., and Kay S.A. 1998. Phytochromes and cryptochromes in the entrainment of the Arabidopsis circadian clock. Science 282: 1488 .

Song S.H., Öztürk N., Denaro T.R., Arat N.O., Kao Y.T., Zhu H., Zhong D., Reppert S.M., and Sancar A. 2007. Formation and function of flavin anion radical in cryptochrome 1 bluelight photoreceptor of monarch butterfly. J. Biol. Chem. 282: 13011.

Stanewsky R., Kaneko M., Emery P., Beretta B., Wager-Smith K., Kay S.A., Rosbash M., and Hall J. C. 1998. The cryb mutation identifies cryptochrome as a circadian photoreceptor in Drosophila. Cell 95: 681.

Swartz T.E., Corchnoy S.B., Christie J.M., Lewis J.W., Szundi I., Briggs W.R., and Bogomolni R.A. 2001. The photocycle of a flavin-binding domain of the blue light photoreceptor phototropin. J. Biol. Chem. 276: 36493.

Thompson C.L., Bowes Rickman C., Shaw S.J., Ebright J.N., Kelly U., Sancar A., and Rickman D.W. 2003. Expression of the blue-light receptor cryptochrome in the human retina. Invest. Ophthalmol. Vis. Sci. 44: 4515.

Thresher R.J., Vitaterna M.H., Miyamoto Y., Kazantsev A., Hsu D.S., Petit C., Selby C.P., Dawut L., Smithies O., Takahashi J.S., and Sancar A. 1998. Role of mouse cryptochrome bluelight photoreceptor in circadian photoresponses. Science 282: 1490.

Todo T., Ryo H., Yamamoto K., Toh H., Inui T., Ayaki H., Nomura T., and Ikenaga M. 1996. Similarity among the Drosophila (6-4)photolyase, a human photolyase homolog, and the DNA photolyase-blue-light photoreceptor family. Science 272: 109.

Todo T., Takemori H., Ryo H., Ihara M., Matsunaga T., Nikaido O., Sato K., and Nomura T. 1993. A new photoreactivating enzyme that specifically repairs ultraviolet light-induced (64)photoproducts. Nature 361: 371.

Tu D.C., Batten M.L., Palczewski K., and Van Gelder R.N. 2004. Non-visual photoreception in the chick iris. Science 306: 129.

Ueda T., Kato A., Kuramitsu S., Terasawa H., and Shimada I. 2005 . Identification and characterization of a second chromophore of DNA photolyase from Thermus thermophilus HB27. J. Biol. Chem. 280: 36237.
Ünsal-Kaçmaz K., Mullen T.E., Kaufmann W.K., and Sancar A. 2005. Coupling of human circadian and cell cycles by the timeless protein. Mol. Cell. Biol. 25: 3109 .

van der Horst G.T., Muijtjens M., Kobayashi K., Takano R., Kanno S., Takao M., de Wit J., Verkerk A., Eker A.P., van Leenen D., Buijs R., Bootsma D., Hoeijmakers J.H., and Yasui A. 1999. Mammalian Cry1 and Cry2 are essential for maintenance of circadian rhythms. Nature 398: 627.

VanVickle-Chavez S.J. and Van Gelder R.N. 2007. Action spectrum of Drosophila cryptochrome. J. Biol. Chem. 282: 10561.

Vitaterna M.H., Selby C.P., Todo T., Niwa H., Thompson C., Fruechte E.M., Hitomi K., Thresher R.J., Ishikawa T., Miyazaki J., Takahashi J.S., and Sancar A. 1999. Differential regulation of mammalian period genes and circadian rhythmicity by cryptochromes 1 and 2. Proc. Natl. Acad. Sci. 96: 12114.

Wang H., Ma L.G., Li J.M., Zhao H.Y., and Deng X.W. 2001. Direct interaction of Arabidopsis cryptochromes with COP1 in light control development. Science 294: 154.

Yang H.Q., Tang R.H., and Cashmore A.R. 2001. The signaling mechanism of Arabidopsis CRY1 involves direct interaction with COP1. Plant Cell 13: 2573.

Yang H.Q., Wu Y.J., Tang R.H., Liu D., Liu Y., and Cashmore A.R. 2000. The C termini of Arabidopsis cryptochromes mediate a constitutive light response. Cell 103: 815.

Yong W., Bao S., Chen S., Li D., Sánchez E.R., and Shuo W. 2007. Mice lacking protein phosphatase 5 are defective in ataxia telangiectasia mutated (ATM)-mediated cell cycle arrest. J. Biol. Chem. 282: 14690.

Young M.W. and Kay S.A. 2001. Time zones: A comparative genetics of circadian clocks. Nat. Rev. Genet. 2: 702.

Yu X., Shalitin D., Liu X., Maymon M., Klejnot J., Yang H., Lopez J., Zhao X., Bendehakkalu K.T., and Lin C. 2007. Derepression of the NC80 motif is critical for the photoactivation of Arabidopsis CRY2. Proc. Natl. Acad. Sci. 104: 7289.

Yuan Q., Metterville D., Briscoe A.D., and Reppert S.M. 2007. Insect cryptochromes: Gene duplication and loss define diverse ways to construct insect circadian clocks. Mol. Biol. Evol. 24: 948.

Zeugner A., Byrdin M., Bouly J.P., Bakrim N., Giovani B., Brettel K., and

Ahmad M. 2005. Light-induced electron transfer in Arabidopsis cryptochrome- 1 correlates with in vivo function. J. Biol. Chem. 280: 19437.

Zhao S. and Sancar A. 1997. Human blue-light photoreceptor hCRY2 specifically interacts with protein serine/threonine phosphatase 5 and modulates its activity. Photochem. Photobiol. 66: 727.

Zhu H., Yuan Q., Briscoe A.D., Froy O., Casselman A., and Reppert S.M. 2005. The two CRYs of the butterfly. Curr. Biol. 15: R953.

Zhong D. 2007. Ultrafast catalytic processes in enzymes. Curr. Opin. Chem. Biol. 11: 174. 


\section{$8_{\mathrm{CSH}}^{\infty} \mathrm{C}$ Cold Spring Harbor Symposia SYMPOSIA on Quantitative Biology}

\section{Structure and Function of Animal Cryptochromes}

N. Öztürk, S.-H. Song, S. Özgür, et al.

Cold Spring Harb Symp Quant Biol 2007 72: 119-131

Access the most recent version at doi:10.1101/sqb.2007.72.015

References This article cites 105 articles, 50 of which can be accessed free at: http://symposium.cshlp.org/content/72/119.full.html\#ref-list-1

\section{License}

Email Alerting

Receive free email alerts when new articles cite this article - sign up in the box at the Service top right corner of the article or click here.

To subscribe to Cold Spring Harbor Symposia on Quantitative Biology go to: http://symposium.cshlp.org/subscriptions 